\title{
ATP-binding cassette transporter A1: A promising therapy target for prostate cancer (Review)
}

\author{
TING XIONG ${ }^{1}$, GANG XU ${ }^{2}$, XUE-LONG HUANG ${ }^{1}$, KAI-QIANG LU ${ }^{1}$, WEI-QUAN XIE ${ }^{1}$, KAI YIN ${ }^{2}$ and JIAN TU ${ }^{1}$ \\ ${ }^{1}$ Institute of Pharmacy and Pharmacology, Hunan Province Cooperative Innovation Center for \\ Molecular Target New Drug Study, and ${ }^{2}$ Department of Diagnostics Teaching and Research, \\ University of South China, Hengyang, Hunan 421001, P.R. China
}

Received May 6, 2017; Accepted November 10, 2017

DOI: $10.3892 / \mathrm{mco} .2017 .1506$

\begin{abstract}
ATP-binding cassette transporter A1 (ABCA1) has been found to mediate the transfer of cellular cholesterol across the plasma membrane to apolipoprotein A-I (apoA-I), and is essential for the synthesis of high-density lipoprotein. Mutations of the ABCA1 gene may induce Tangier disease and familial hypoalphalipoproteinemia; they may also lead to loss of cellular cholesterol homeostasis in prostate cancer, and increased intracellular cholesterol levels are frequently found in prostate cancer cells. Recent studies have demonstrated that ABCA1 may exert anticancer effects through cellular cholesterol efflux, which has been attracting increasing attention in association with prostate cancer. The aim of the present review was to focus on the current views on prostate cancer progression and the various functions of $\mathrm{ABCA} 1$, in order to provide new therapeutic targets for prostate cancer.
\end{abstract}

Correspondence to: Dr Jian Tu, Institute of Pharmacy and Pharmacology, Hunan Province Cooperative Innovation Center for Molecular Target New Drug Study, University of South China, 28 Changsheng Road, Hengyang, Hunan 421001, P.R. China E-mail: jian.tu@cshs.org

Abbreviations: ABC, ATP-binding cassette; LDL, low-density lipoprotein; apoA-I, apolipoprotein A-I; TGF- $\beta$, transforming growth factor- $\beta$; JAK2, Janus kinase 2 ; STAT3, signal transducer and activator of transcription 3; HDL, high-density lipoprotein; LXR, liver X receptor; MDR, multidrug resistance; PI3K, phosphoinositide 3-kinase; ERK, extracellular signal-regulated kinase; P-gp, P-glycoprotein; MRP, multidrug resistance-associated protein; EGFR, epidermal growth factor receptor

Key words: ATP-binding cassette transporter A1, prostate cancer, cholesterol

\section{Contents}

1. Introduction

2. Effect of ABCA1 on the progression of prostate cancer

3. Association of ABCA1 with apoptosis and autophagy in prostate cancer

4. ABCA1 and multidrug resistance (MDR)

\section{Introduction}

Prostate cancer is a common malignancy among men in Western countries; however, a sharp increase in its morbidity and mortality was recently observed in several Asian countries, including China (1). Patients with aggressive pathological characteristics are at increased risk for tumor progression and metastasis, even following radical treatment. Furthermore, these characteristics are commonly found in tumors from patients who succumb to prostate cancer. Cholesterol homeostasis is crucial for cell function and survival, whereas dysregulation of cholesterol homeostasis is known to be associated with multiple cancers, including prostate cancer (2), and altered lipid metabolism is increasingly recognized as a hallmark of prostate cancer cells (3). In fact, lethal prostate cancers exhibit higher expression of squalene monooxygenase, which is the second rate-limiting enzyme of cholesterol synthesis (4). Moreover, low serum cholesterol levels have been found in prostate cancer patients, suggesting that cholesterol may accumulate in tumor tissue. Compared with normal prostate cells, prostate tumor cells exhibit increased levels of intracellular cholesterol precursors, with loss of ABCA1-mediated cholesterol efflux (5).

ATP-binding cassette (ABC) transporters are transmembrane proteins responsible for the transfer of various substrates through extracellular and intracellular membranes. The ABC-type transporters act as gatekeepers by allowing or limiting the entrance of a wide variety of substrates across cellular membranes (6). A total of 51 ABC transporter genes have been identified and are grouped into seven subfamilies, namely A-G, based on their phylogenetic distance (7). The physiological importance of ABCA subfamily proteins is underscored by their association with various inherited diseases. Examples of ABC A-subfamily disorders include Tangier disease (ABCA1), Alzheimer's disease 
(ABCA2/ABCA7), Stargardt's disease (ABCR/ABCA4), harlequin-type ichthyosis (ABCA12) and others (8) (Table I). ABCA1, an ABC subfamily A exporter, mediates the cellular efflux of phospholipids and cholesterol to the extracellular acceptor apolipoprotein A-I (apoA-I) for generation of nascent high-density lipoprotein (HDL) (9). The human ABCA1 has a length of 2,261 amino acids, and was found to contain two transmembrane domains (TMDs) (10). Each TMD is followed by a cytoplasmic region comprising one nucleotide-binding domain (NBD) and one small regulatory domain. The NBDs and regulatory domains are the most highly conserved elements among the ABCA subfamily. The structure of ABCA1 reveals a polar cluster on one side of TMD1 close to the intracellular boundary (11). The structure of the ABCA1 presented herein represents a major step towards the mechanistic understanding of ABCA1-mediated lipid export and nascent HDL biogenesis (12). ABCA1 has been shown to be necessary for the synthesis of HDL by exporting cholesterol out of the cells (13). Functional ABCA1 mediates the expansion of the N-terminus of apoA-I on the cell surface, followed by the release of nascent HDL, which is the only path for elimination of cholesterol from the body (14). Two missense mutations of human ABCA1 proteins linked to Tangier disease and familial HDL deficiency were previously found to be associated with diminished cholesterol efflux activity, and the functionality of these ABCA1 mutants in terms of tumor inhibition and cholesterol efflux was tested in prostate cancer cells lacking endogenous ABCA1 expression (15). Over 50 mutations in the ABCA1 gene were recently identified. The human ABCA1 has been shown to be closely associated with the development of various human cancers, including prostate, colon and breast cancer (16-18). Prostate cancer patients with higher serum low-density lipoprotein (LDL) levels exhibited significantly shorter overall survival. Low ABCA1 expression has also been associated with shorter survival in patients with prostate cancer. This may be explained by the use of lipid as an energy source during growth and metastasis of prostate cancer cells. ABCA1 has been shown to play a critical role in the synthesis of HDL particles by exporting cellular cholesterol $(19,20)$.

Considering these findings, further investigation into ABCA1 as a tumor suppressor in prostate cancer is required. We herein review the role of ABCA1 in prostate cancer and the ongoing research on its association with cancer proliferation, invasion and migration, apoptosis and multidrug resistance. The aim of the present review was to demonstrate that ABCA1 represents a promising new target in the treatment of prostate cancer.

\section{Effect of ABCA1 on the progression of prostate cancer}

The ABCA1 protein mediates cellular cholesterol transfer to apoA-I through the plasma membrane. ABCA1 has been found to play a crucial role in the etiology of various neurological and cardiovascular diseases, including inflammation and metabolic syndrome (21), and may also be involved in the initiation and development of prostate cancer. A causal association between ABCA1-mediated tumor inhibition and mitochondrial cholesterol depletion has been identified through testing the dependence of ABCA1 anticancer activity on its efflux capacity (22). In addition, transforming growth factor (TGF)- $\beta$ signaling plays a key role in prostate cancer occurrence and maintenance of cancer stem cell characteristics. The growth inhibitory effect of TGF- $\beta$ may be due to overexpression of ABCA1 in prostate cancer cells (23). The expression of ABCA1 is associated with nuclear receptor of liver $\mathrm{X}$ receptor (LXR) and LXR forms a heterodimer with retinoid X receptor and regulates transcription of ABCA1 by binding to the DR-4 promoter element (24). Furthermore, TGF- $\beta$ may increase ABCA1 expression through activation of LXR signaling. It has been demonstrated that LXR activation, with accompanying upregulation of ABCA1 expression, may decrease cholesterol levels and reduce the growth of prostate cancer cell xenograft tumors in mice (25). Furthermore, LXR agonists have been shown to induce expression of ABCA1, inhibit tumor growth and reduce progression to androgen independence in a xenograft model of prostate cancer (26). The antitumor effects of these compounds may involve a variety of mechanisms: Statins inhibit GTPases such as Ras and Rho family proteins via blocking protein prenylation and/or farnesylation, and LXR agonists induce cell cycle arrest through upregulation of p27. Conversely, intracellular cholesterol promotes prostate cancer progression as a substrate for de novo androgen synthesis and through regulation of Akt signaling (22).

Hypermethylation of the ABCA1 promoter has been shown to silence ABCA1 expression and is associated with high-grade prostate cancer (27). The interaction of apoA-I with ABCA1 activates the signaling molecule Janus kinase 2 (JAK2), which optimizes the cholesterol efflux activity of ABCA1 by phosphorylation (28). ABCA1-mediated JAK2 activation also activates signal transducer and activator of transcription 3 (STAT3), which significantly attenuates the expression of proinflammatory cytokines in macrophages. The anti-inflammatory effects of the apoA-I/ABCA1/STAT3 pathway, however, is dependent on the activity of suppressor of cytokine signaling 3. Taken together, these findings suggest that the interaction of apoA-I/ABCA1 activates cholesterol efflux and STAT3 branching pathways to synergistically inhibit inflammation in macrophages (29). It was also demonstrated that the apoA-I/ABCA1 interaction with the JAK2/STAT3 pathway to inhibit macrophage inflammatory responses is independent of $\mathrm{ABCA} 1$ lipid transport function. ABCA1 mutation carriers also exhibit an increased incidence of systemic and plaque inflammation (30). It has been observed that aberrant regulation of tristetraprolin and human antigen $\mathrm{R}$ plays an important role in the progression of prostate cancer and other inflammation-related cancers, as well as in cancer cell proliferation, apoptosis, angiogenesis, invasion and chemoresistance (31).

Epidemiological data have associated a reduced risk of prostate cancer with low serum cholesterol, as well as with the use of statins, demonstrating the role of cholesterol metabolism in the development of aggressive prostate cancer (32). The efflux of intracellular cholesterol is mediated by reverse cholesterol transporters, such as scavenger receptor class B type I, a membrane receptor with extensive tissue distribution and a number of physiological functions (33). A reduction in Akt-dependent survival signaling may also contribute to the anticancer activity of ABCA1. In particular, $\sim 70 \%$ of advanced PCa exhibits loss of phosphatase and tensin homolog (PTEN) 
Table I. Structure and function of ABCA subfamily proteins.

\begin{tabular}{ll} 
Proteins & \multicolumn{1}{c}{ Structure } \\
\hline ABCA1 & Length, 2,261 amino acids; contains two \\
& TMDs, and a polar cluster on one side of \\
& TMD1 close to the intracellular boundary.
\end{tabular}

ABCA2 Coding region $7.3 \mathrm{~kb}$ in size, codes for a 2,436-amino acid polypeptide, comprises 48 exons.

ABCA3 Consists of 33 exons encoding a 1,704-amino acid $(\sim 150 \mathrm{kDa})$ protein.

ABCA4 Transcribes a large retina-specific protein with two TMDs, two glycosylated ECDs and two NBDs.

ABCA5 Consists of 1,642 amino acid residues, with two sets of six transmembrane segments and an NBD.

ABCA7 Spans a region of $\sim 32 \mathrm{~kb}$ and comprises 46 exons.

ABAC9 Consists of 39 exons, genomic region of $\sim 85 \mathrm{~kb}$ chromosome $17 \mathrm{q} 24.2$.

ABCA10 The coding sequence of ABCA10 is $4.6 \mathrm{~kb}$ in size and codes for a 1,543-amino acid protein.

ABCA12 Located on the long (q) arm of chromosome 2 between positions 34 and 35 .

ABCA13 Coding region is $6.7 \mathrm{~kb}$ in size and encodes a protein consisting of 2,143 amino acids with a predicted molecular weight of $240 \mathrm{kDa}$.
Function

(Refs.)

Synthesis of HDL, exporting cholesterol out of the cells, lipid efflux activity. Mutation of the ABCA1 gene may induce Tangier disease and familial hypoalphalipoproteinemia. Tumor suppressor function in cancer.

Highly expressed in brain tissue; may play a role

in macrophage lipid metabolism and neural development.

Mutation of the ABCA3 gene is the most common cause of surfactant deficiency and is associated with cataract-microcornea syndrome.

An inward-directed retinoid flippase; mutation of ABCA4 induces Stargardt's disease.

Mutations in ABCA5 cause hair overgrowth and may be associated with a lysosomal disease, particularly in cardiomyocytes and follicular cells.

Predisposes to Alzheimer's disease.

Involved in monocyte differentiation and macrophage lipid homeostasis.

Involved in macrophage lipid homeostasis.

Active in some types of skin cells, linked to harlequin-type ichthyosis.

Linked to psychiatric disorders such as

ABC, ATP-binding cassette; TMD, transmembrane domain; HDL, high-density lipoprotein; ECD, extracellular domain; NBD, nucleotide-binding domain.

or consequent activation of the phosphoinositide 3-kinase (PI3K)/Akt pathway, which leads to enhanced cell survival, migration and castration-resistant growth (40).

Intracellular cholesterol promotes prostate cancer progression, as it is a substrate for de novo androgen synthesis, and by regulation of Akt signaling. Akt increased the expression of ABCA1 by delaying its degradation, but does not alter the mRNA levels (41). The protective effect of the ABCA1 protein is mediated by Akt2, possibly by stabilizing ABCA1 resistance to calpain-mediated proteolysis. ABCA1 reduces LDL receptor expression, decreases intracellular cholesterol and relies on LXR $\alpha$. Of note, the epidermal growth factor receptor (EGFR) signal opposes the effects of LXR $\alpha$ on cholesterol homeostasis, whereas EGFR inhibitors interact with LXR $\alpha$ agonists to destroy cancer cells. Inhibition of activation of LXR $\alpha$ by sterol metabolites may be an effective strategy for targeting EGFR-KRAS signaling against cancer (42).

The promoter region of the ABCA1 gene has been shown to be epigenetically silenced by hypermethylation in the LNCaP human prostate cancer cell line. This suggests that loss of ABCA1 expression may lead to a significant alteration in cholesterol homeostasis and may indicate a fundamental association between high circulating cholesterol levels and prostate cancer growth (43). Epidemiological studies have identified a correlation between high serum cholesterol levels and prostate cancer, as well as a protective effect of statin use (44).

In conclusion, hypermethylation and gene inactivation of ABCA1 promoters leads to accumulation of cholesterol in prostate cancer cells. Thus, this cellular cholesterol efflux pathway may be an important determinant of prostate cancer aggressiveness, as well as a potential therapeutic target.

\section{Association of ABCA1 with apoptosis and autophagy in prostate cancer}

Overexpression of ABCA1 leads to decreased cellular cholesterol and tumor growth inhibition in prostate cancer cells. ABCA1 antitumor activity requires an efflux function and appears to be mediated by reduced mitochondrial cholesterol combined with an increased potential for release of cell death-promoting 


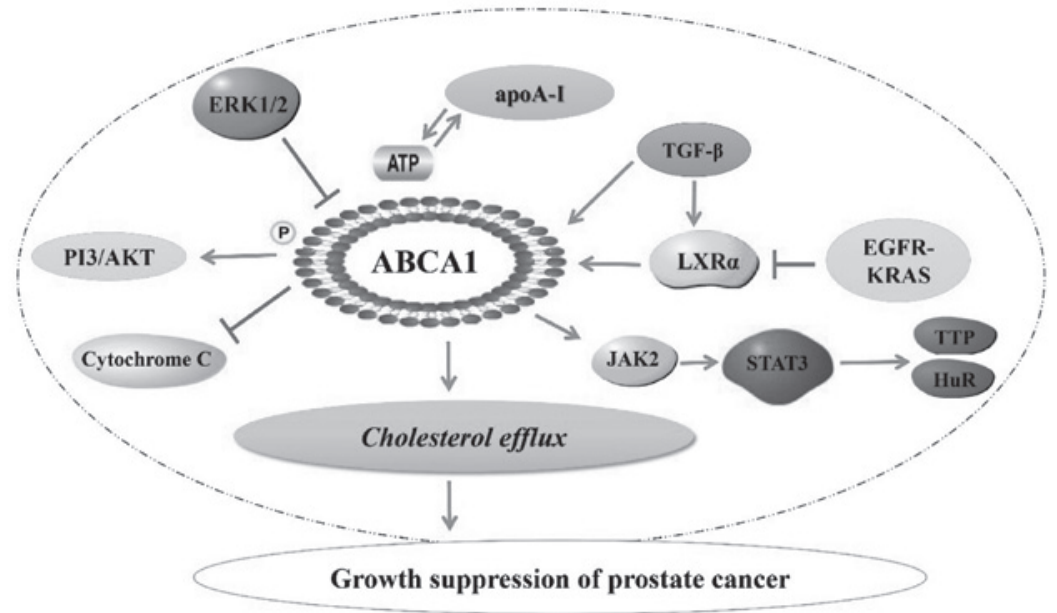

Figure 1. Association of ABCA1 with prostate cancer. ABCA1, ATP-binding cassette transporter A1; PI3, phosphoinositide 3 kinase; ERK, extracellular signal-regulated kinase; apoA-I, apolipoprotein A-I; TGF, transforming growth factor; LXR, liver X receptor; EGFR, epidermal growth factor receptor; JAK2, Janus kinase 2; STAT3, signal transducer and activator of transcription 3; TTP, tristetraprolin; HuR, human antigen R.

molecules, such as cytochrome $\mathrm{C}$, from mitochondria. The loss-of-function phenotype for somatic mutations identified in human colon cancer suggests the role of ABCA1 as a tumor suppressor, albeit in the context of increased cholesterol synthesis sufficient to raise the levels of mitochondrial cholesterol. This antitumor effect may be due to apoptosis, as overexpression of ABCA1 has been shown to increase mitochondrial release of cytochrome $\mathrm{C}$ in vitro and in vivo (45).

Furthermore, downregulation of ABCA1 is present in most prostate cancer specimens, and ABCA1 expression levels are inversely associated with Gleason score. DNA hypermethylation on the ABCA1 promoter in LNCaP cells effectively inhibits basal expression and prevents the complete induction of trans-activators. The loss of ABCA1 protein expression directly results in high intracellular cholesterol levels, thereby contributing to an environment conducive to tumor progression. In addition, ABCA1 hypermethylation was exclusively detected in intermediate- and high-grade prostate cancer. Thus, epigenetic inactivation of ABCA1 may be intimately associated with prostate cancer progression, suggesting that dysregulation of cellular cholesterol levels in prostate epithelial cells creates an environment conducive to tumor progression.

Autophagy is an intracellular protein-degradation process that is conserved across eukaryotes, including yeast and humans, which responds to cellular stress conditions to maintain a healthy cellular status by degrading and recycling cytoplasmic contents via the lysosomal route (46). Under nutrient starvation conditions, intracellular proteins are transported to lysosomes and vacuoles via membranous structures known as autophagosomes, and are degraded. It has been demonstrated that autophagy participates in the regulation of lipid metabolism and cholesterol homeostasis, with a special emphasis on macrophage-derived foam cells. Autophagy has emerged as an alternative lipid metabolic pathway through the lysosomal degradative pathway, making this process a potential therapeutic target for diseases associated with disorders of lipid metabolism (47). Of note, all these lipoproteins increase autophagic flux in macrophages, and autophagy depletion in these cells results in dimin- ished cholesterol efflux to apoA-I. The autophagy inhibitor 3-methyladenine and an ATG5 siRNA were found to significantly attenuate autophagy, subsequently suppressing the ABCA1-mediated cholesterol efflux (48). Biochemical studies have demonstrated that the accumulation of cholesterol esters in prostate cancer cells is the result of loss of the tumor suppressor PTEN and activation of the PI3K/Akt pathway. AMP-activated protein kinase (AMPK)-dependent ABCA1 expression also involves the mammalian target of rapamycin (mTOR) pathway and inhibits the extracellular-signal regulated kinase (ERK). AMPK-induced ABCA1 expression leads to cholesterol efflux from human macrophages (49). Through the ERK1/2 pathway, intracellular ABCA1 exerts a protective effect against oxidative stress caused by ischemia. The PI3K/Akt/mTOR signaling pathway is a classic autophagy pathway. PI3K and Akt suppression may inhibit mTOR phosphorylation at the $\operatorname{Ser}^{2448}$ site, thereby enhancing the expression of autophagy-related proteins and inducing autophagy (50).

It has been demonstrated that elevated mitochondrial cholesterol promotes resistance to cell death in prostate cancer and may lead to malignant cell transformation (51). Inhibitory RNA (RNAi) knockdown of squalene synthase, the enzyme which catalyzes the first committed step of cholesterol synthesis, leads to decreased cell proliferation and survival of prostate cancer cells in vitro (52). ABCA1 promotes a reduction in mitochondrial cholesterol levels, mitochondrial permeability transition-associated cytochrome $\mathrm{C}$ release, and non-apoptotic/necrotic cell death in response to cell stress. Ultimately, ABCA1-mediated cell death depends on cholesterol for modulation of mitochondrial function, while ABCA1 deficiency allows for elevated mitochondrial cholesterol and ultimately promotes cancer cell survival.

\section{ABCA1 and multidrug resistance (MDR)}

MDR is a major cause of failure of prostate cancer chemotherapy and is associated with an increased mortality risk (53). The PI3K/Akt signaling cascade may be correlated with MDR1/P-glycoprotein (P-gp) and plays a critical role in 
the MDR phenotype. MDR-associated protein (MRP) 1 is expressed in a variety of human malignancies (54). ABCA1 may export anticancer drugs and decrease intracellular drug concentration, which is associated with limited success of anticancer chemotherapy, attributed to the the cross-resistance of tumor cells and referred to as MDR. The role of ABCA1 in MDR has been well-characterized, and measuring the expression of ABCA1 may be used for predicting the response to anticancer drugs.

Cell viability in prostate cancer may be regulated by modifying critical pathways mediating novel androgen signaling by AR-targeting microRNAs. In this study, androgens stimulating miR-19a, and miR-19a directly repressing ABCA1 mRNA expression, may represent a possible mechanism underlying androgen-mediated repression of $\mathrm{ABCA} 1$, promoting $\mathrm{PCa}$ cell proliferation (55). The activation of ABCA1 was also shown to inhibit the proliferation of androgen-dependent prostate cancer cells, and androgen treatment in LNCaP cells significantly inhibited the expression of ABCA1 mRNA (56). In vitro studies demonstrated that $\mathrm{LXR}$ agonists activate transcription of the MRP2 gene to promote excretion of endogenous and xenobiotic compounds from hepatocytes into the bile (57).

ABCA1 also transfers phospholipids, preferentially phosphatidylcholine and cholesterol, to lipid-free apoA-I to produce pre- $\beta$-HDL, which also binds directly to MDR 1 and regulates substrate recognition via MDR1 (58). Eukaryotic $\mathrm{ABC}$ proteins may retain similar substrate binding pockets and move substrates in an ATP-dependent manner. The prototype of eukaryote $\mathrm{ABC}$ proteins may be those involved in membrane lipid transport.

If overexpression of ABCA1 promotes MDR in tumors, ABCA1 gene mutations may lead to drug resistance and alter substrate specificity. Thus, the study of ABCA1 gene mutations and their role in MDR is crucial for individualized patient treatment.

In conclusion, ABCA1 plays an important role in the prevention and treatment of prostate cancer through regulation of cellular cholesterol efflux (Fig. 1). The association of lower ABCA1 expression with shorter survival in prostate cancer prompts ongoing research to further elucidate the mechanism underlying ABCA1 regulation of tumor growth. Various cross-regulatory mechanisms may be the focus of future research. Studies on ABCA1 will contribute to the understanding of drug transport and the role of cholesterol in cancer growth, and may lead to optimization of treatment for prostate and other cancers. Additionally, the study of ABCA1 gene mutations and associated differences in expression and functionality may lay the foundation for future pharmacogenetics in prostate cancer treatment. Ultimately, studies in ABCA1 may provide a novel therapeutic target in cancer.

\section{Acknowledgements}

The present study was funded by the National Natural Science Foundation of China (grant no. 81541163); the Natural Science Foundation of Hunan Province (grant no. 2015JJ3101); the Open Fund Based on Innovation Platform of Hunan Provincial Education Department (grant no. 15K111); the Hunan Provincial Cooperative Innovation Center for Molecular Target
New Drug Study (grant no. 2014-405); and the Innovation Program of Graduate Education in Cooperative Innovation Center for Molecular Target New Drug of University of South China (grant no. 0223-0002-00028).

\section{References}

1. Zong Y, Goldstein AS and Huang J: The molecular basis for ethnic variation and histological subtype differences in prostate cancer. Sci China Life Sci 56: 780-787, 2013.

2. Adlakha YK, Khanna S, Singh R, Singh VP, Agrawal A and Saini N: Pro-apoptotic miRNA-128-2 modulates ABCA1, ABCG1 and RXR $\alpha$ expression and cholesterol homeostasis. Cell Death Dis 4: e780, 2013.

3. Yue S, Li J, Lee SY, Lee HJ, Shao T, Song B, Cheng L, Masterson TA, Liu X, Ratliff TL and Cheng JX: Cholesteryl ester accumulation induced by PTEN loss and PI3K/AKT activation underlies human prostate cancer aggressiveness. Cell Metab 19: 393-406, 2014.

4. Stopsack KH, Gerke TA, Andrén O, Andersson SO, Giovannucci EL, Mucci LA and Rider JR: Cholesterol uptake and regulation in high-grade and lethal prostate cancers. Carcinogenesis 38: 806-811, 2017.

5. Cruz PM, Mo H, McConathy WJ, Sabnis N and Lacko AG: The role of cholesterol metabolism and cholesterol transport in carcinogenesis: A review of scientific findings, relevant to future cancer therapeutics. Front Pharmacol 4: 119, 2013.

6. Flores K, Manautou JE and Renfro JL: Gender-specific expression of ATP-binding cassette (Abc) transporters and cytoprotective genes in mouse choroid plexus. Toxicology 386: 84-92, 2017.

7. Durmus S, Hendrikx JJ and Schinkel AH: Apical ABC transporters and cancer chemotherapeutic drug disposition. Adv Cancer Res 125: 1-41, 2015.

8. Kaminski WE, Piehler A and Wenzel JJ: ABC A-subfamily transporters: Structure, function and disease. Biochim Biophys Acta 1762: 510-524, 2006.

9. Qian H, Zhao X, Cao P, Lei J, Yan N and Gong X: Structure of the human lipid exporter ABCA1. Cell 169: 1228-1239.e10, 2017.

10. Fitzgerald ML, Mendez AJ, Moore KJ, Andersson LP, Panjeton HA and Freeman MW: ATP-binding cassette transporter A1 contains an NH2-terminal signal anchor sequence that translocates the protein's first hydrophilic domain to the exoplasmic space. J Biol Chem 276: 15137-15145, 2001.

11. Lamping E, Baret PV, Holmes AR, Monk BC, Goffeau A and Cannon RD: Fungal PDR transporters: Phylogeny, topology, motifs and function. Fungal Genet Biol 47: 127-142, 2010.

12. Lund-Katz S and Phillips MC: High density lipoprotein structure-function and role in reverse cholesterol transport. Subcell Biochem 51: 183-227, 2010.

13. Sene A, Khan AA, Cox D, Nakamura RE, Santeford A, Kim BM, Sidhu R, Onken MD, Harbour JW, Hagbi-Levi S, et al: Impaired cholesterol efflux in senescent macrophages promotes age-related macular degeneration. Cell Metab 17: 549-561, 2013.

14. Wang S and Smith JD: ABCA1 and nascent HDL biogenesis. Biofactors 40: 547-554, 2014.

15. Murano T, Yamaguchi T, Tatsuno I, Suzuki M, Noike H, Takanami T, Yoshida T, Suzuki M, Hashimoto R, Maeno T, et al: Subfraction analysis of circulating lipoproteins in a patient with Tangier disease due to a novel ABCA1 mutation. Clin Chim Acta 452: 167-172, 2016.

16. Bi DP, Yin $\mathrm{CH}$, Zhang XY, Yang NN and Xu JY: MiR-183 functions as an oncogene by targeting ABCA1 in colon cancer. Oncol Rep 35: 2873-2879, 2016.

17. Sharma M, Tuaine J, McLaren B, Waters DL, Black K, Jones LM and McCormick SP: Chemotherapy agents alter plasma lipids in breast cancer patients and show differential effects on lipid metabolism genes in liver cells. PLoS One 11: e0148049, 2016.

18. Kaneko T, Kanno C, Ichikawa-Tomikawa N, Kashiwagi K, Yaginuma N, Ohkoshi C, Tanaka M, Sugino T, Imura T, Hasegawa $\mathrm{H}$ and Chiba $\mathrm{H}$ : Liver $\mathrm{X}$ receptor reduces proliferation of human oral cancer cells by promoting cholesterol efflux via up-regulation of ABCA1 expression. Oncotarget 6: 33345-33357, 2015.

19. Sviridov DO, Drake SK, Freeman LA and Remaley AT: Amphipathic polyproline peptides stimulate cholesterol efflux by the ABCA1 transporter. Biochem Biophys Res Commun 471: 560-565, 2016. 
20. Zhang M, Li L, Xie W, Wu JF, Yao F, Tan YL, Xia XD, Liu XY, Liu D, Lan G, et al: Apolipoprotein A-1 binding protein promotes macrophage cholesterol efflux by facilitating apolipoprotein A-1 binding to ABCA1 and preventing ABCA1 degradation. Atherosclerosis 248: 149-159, 2016.

21. Sultana A, Cochran BJ, Tabet F, Patel M, Torres LC, Barter PJ and Rye KA: Inhibition of inflammatory signaling pathways in 3T3-L1 adipocytes by apolipoprotein A-I. FASEB J 30: $2324-2335,2016$

22. Smith B and Land H: Anticancer activity of the cholesterol exporter ABCA1 gene. Cell Rep 2: 580-590, 2012.

23. Her NG, Jeong SI, Cho K, Ha TK, Han J, Ko KP, Park SK, Lee JH, Lee MG, Ryu BK and Chi SG: PPARd promotes oncogenic redirection of TGF- $\beta 1$ signaling through the activation of the ABCA1-Cav1 pathway. Cell Cycle 12: 1521-1535, 2013.

24. Tazoe F, Yagyu H, Okazaki H, Igarashi M, Eto K, Nagashima S Inaba T, Shimano H, Osuga $\mathbf{J}$ and Ishibashi S: Induction of ABCA1 by overexpression of hormone-sensitive lipase in macrophages. Biochem Biophys Res Commun 376: 111-115, 2008.

25. Dufour J, Viennois E, De Boussac H, Baron S and Lobaccaro JM: Oxysterol receptors, AKT and prostate cancer. Curr Opin Pharmacol 12: 724-728, 2012.

26. Chuu CP, Hiipakka RA, Kokontis JM, Fukuchi J, Chen RY and Liao S: Inhibition of tumor growth and progression of LNCaP prostate cancer cells in athymic mice by androgen and liver $\mathrm{X}$ receptor agonist. Cancer Res 66: 6482-6486, 2006.

27. Lee BH, Taylor MG, Robinet P, Smith JD, Schweitzer J, Sehayek E, Falzarano SM, Magi-Galluzzi C, Klein EA and Ting AH: Dysregulation of cholesterol homeostasis in human prostate cancer through loss of ABCA1. Cancer Res 73: 1211-1218, 2013.

28. Zhao GJ, Yin K, Fu YC and Tang CK: The interaction of ApoA-I and ABCA1 triggers signal transduction pathways to mediate efflux of cellular lipids. Mol Med 18: 149-158, 2012.

29. Tang C, Houston BA, Storey C and LeBoeuf RC: Both STAT3 activation and cholesterol efflux contribute to the anti-inflammatory effect of apoA-I/ABCA1 interaction in macrophages J Lipid Res 57: 848-857, 2016.

30. Bochem AE, van der Valk FM, Tolani S, Stroes ES, Westerterp M and Tall AR: Increased systemic and plaque inflammation in ABCA1 mutation carriers with attenuation by statins. Arterioscler Thromb Vasc Biol 35: 1663-1669, 2015.

31. Ramírez CM, Lin CS, Abdelmohsen K, Goedeke L, Yoon JH, Madrigal-Matute J, Martin-Ventura JL, Vo DT, Uren PJ, Penalva LO, et al: RNA binding protein HuR regulates the expression of ABCA1. J Lipid Res 55: 1066-1076, 2014.

32. Ohno Y, Ohori M, Nakashima J, Okubo H, Satake N, Hashimoto T and Tachibana M: Association between preoperative serum total cholesterol level and biochemical recurrence in prostate cancer patients who underwent radical prostatectomy. Mol Clin Oncol 4: 1073-1077, 2016

33. Cardenas E and Ghosh R: Vitamin E: A dark horse at the crossroad of cancer management. Biochem Pharmacol 86 845-852, 2013.

34. Kaminski WE, Piehler A, Püllmann K, Porsch-Ozcürümez M, Duong C, Bared GM, Büchler C and Schmitz G: Complete coding sequence, promoter region, and genomic structure of the human ABCA2 gene and evidence for sterol-dependent regulation in macrophages. Biochem Biophys Res Commun 281: 249-258, 2001

35. Paolini A, Baldassarre A, Del Gaudio I and Masotti A: Structural features of the ATP-Binding Cassette (ABC) transporter ABCA3. Int J Mol Sci 16: 19631-19644, 2015.

36. Kubo Y, Sekiya S, Ohigashi M, Takenaka C, Tamura K, Nada S, Nishi T, Yamamoto A and Yamaguchi A: ABCA5 resides in lysosomes, and ABCA5 knockout mice develop lysosomal disease-like symptoms. Mol Cell Biol 25: 4138-4149, 2005.

37. Broccardo C, Osorio J, Luciani MF, Schriml LM, Prades C, Shulenin S, Arnould I, Naudin L, Lafargue C, Rosier M, et al: Comparative analysis of the promoter structure and genomic organization of the human and mouse $\mathrm{ABCA} 7$ gene encoding a novel ABCA transporter. Cytogenet Cell Genet 92: 264-270, 2001.

38. Piehler A, Kaminski WE, Wenzel JJ, Langmann T and Schmitz G: Molecular structure of a novel cholesterol-responsive A subclass ABC transporter, ABCA9. Biochem Biophys Res Commun 295: 408-416, 2002

39. Maeß MB, Stolle K, Cullen P and Lorkowski S: Evidence for an alternative genomic structure, mRNA and protein sequence of human ABCA13. Gene 515: 298-307, 2013.
40. Marques RB, Aghai A, de Ridder CMA, Stuurman D, Hoeben S, Boer A, Ellston RP, Barry ST, Davies BR, Trapman J and van Weerden WM: High efficacy of combination therapy Using PI3K/AKT inhibitors with androgen deprivation in prostate cancer preclinical models. Eur Urol 67: 1177-1185, 2015.

41. Okoro EU, Guo Z and Yang H: Akt isoform-dependent regulation of ATP-Binding cassette A1 expression by apolipoprotein E. Biochem Biophys Res Commun 477: 123-128, 2016.

42. Gabitova L, Restifo D, Gorin A, Manocha K, Handorf E, Yang DH, Cai KQ, Klein-Szanto AJ, Cunningham D, Kratz LE, et al: Endogenous sterol metabolites regulate growth of EGFR/KRAS-dependent tumors via LXR. Cell Rep 12: 1927-1938, 2015

43. Solomon KR, Allott EH, Freeman MR and Freedland SJ: Words of wisdom. Re: Dysregulation of cholesterol homeostasis in human prostate cancer through loss of ABCA1. Eur Urol 63: 1128-1129, 2013.

44. Hayashi N, Matsushima M, Yamamoto T, Sasaki H, Takahashi H and Egawa S: The impact of hypertriglyceridemia on prostate cancer development in patients aged $\geq 60$ years. BJU Int 109: 515-519, 2012

45. Chou JL, Huang RL, Shay J, Chen LY, Lin SJ, Yan PS, Chao WT, Lai YH, Lai YL, Chao TK, et al: Hypermethylation of the TGF- $\beta$ target, ABCA1 is associated with poor prognosis in ovarian cancer patients. Clin Epigenetics 7: 1,2015.

46. He C and Klionsky DJ: Regulation mechanisms and signaling pathways of autophagy. Annu Rev Genet 43: 67-93, 2009.

47. Puri $\mathrm{P}$ and Chandra A: Autophagy modulation as a potential therapeutic target for liver diseases. J Clin Exp Hepatol 4: 51-59, 2014.

48. Han XB, Li HX, Jiang YQ, Wang H, Li XS, Kou JY, Zheng YH, Liu ZN, Li H, Li J, et al: Upconversion nanoparticle-mediated photodynamic therapy induces autophagy and cholesterol efflux of macrophage-derived foam cells via ROS generation. Cell Death Dis 8: e2864, 2017.

49. Lin XL, Liu MH, Hu HJ, Feng HR, Fan XJ, Zou WW, Pan YQ, $\mathrm{Hu} \mathrm{XM}$ and Wang Z: Curcumin enhanced cholesterol efflux by upregulating ABCA1 expression through AMPK-SIRT1-LXRa signaling in THP-1 macrophage-derived foam cells. DNA Cell Biol 34: 561-572, 2015.

50. Jiang Y, Kou J, Han X, Li X, Zhong Z, Liu Z, Zheng Y, Tian Y and Yang L: ROS-dependent activation of autophagy through the $\mathrm{PI} 3 \mathrm{~K} / \mathrm{Akt} / \mathrm{mTOR}$ pathway is induced by hydroxysafflor yellow A-sonodynamic therapy in THP-1 macrophages. Oxid Med Cell Longev 2017: 8519169, 2017.

51. Wu X, Daniels G, Lee P and Monaco ME: Lipid metabolism in prostate cancer. Am J Clin Exp Urol 2: 111-120, 2014.

52. Brusselmans K, Timmermans L, Van de Sande $T$, Van Veldhoven PP, Guan G, Shechter I, Claessens F, Verhoeven G and Swinnen JV: Squalene synthase, a determinant of Raft-associated cholesterol and modulator of cancer cell proliferation. J Biol Chem 282: 18777-18785, 2007.

53. Wang H, Jia XH, Chen JR, Yi YJ, Wang JY, Li YJ and Xie SY: HOXB4 knockdown reverses multidrug resistance of human myelogenous leukemia K562/ADM cells by downregulating P-gp, MRP1 and BCRP expression via PI3K/Akt signaling pathway. Int J Oncol 49: 2529-2537, 2016.

54. Chen JR, Jia XH, Wang H, Yi YJ, Wang JY and Li YJ: Timosaponin A-III reverses multi-drug resistance in human chronic myelogenous leukemia K562/ADM cells via downregulation of MDR1 and MRP1 expression by inhibiting PI3K/Akt signaling pathway. Int J Oncol 48: 2063-2070, 2016.

55. Mo W, Zhang J, Li X, Meng D, Gao Y, Yang S, Wan X, Zhou C, Guo F, Huang Y, et al: Identification of novel AR-targeted microRNAs mediating androgen signalling through critical pathways to regulate cell viability in prostate cancer. PLoS One 8: e56592, 2013.

56. Sekine Y, Demosky SJ, Stonik JA, Furuya Y, Koike H, Suzuki K and Remaley AT: High-density lipoprotein induces proliferation and migration of human prostate androgen-independent cancer cells by an ABCA1-dependent mechanism. Mol Cancer Res 8: 1284-1294, 2010

57. Chisaki I, Kobayashi M, Itagaki S, Hirano T and Iseki K: Liver X receptor regulates expression of MRP2 but not that of MDR1 and BCRP in the liver. Biochim Biophys Acta 1788: 2396-2403, 2009.

58. Kimura Y, Morita SY, Matsuo M and Ueda K: Mechanism of multidrug recognition by MDR1/ABCB1. Cancer Sci 98: 1303-1310, 2007. 